\title{
The Problem of Image Recovery by the Metric Projections in Banach Spaces
}

\author{
Yasunori Kimura $^{1}$ and Kazuhide Nakajo ${ }^{2}$ \\ ${ }^{1}$ Department of Information Science, Toho University, Miyama, Funabashi, Chiba 274-8510, Japan \\ ${ }^{2}$ Sundai Preparatory School, Kanda-Surugadai, Chiyoda-ku, Tokyo 101-8313, Japan \\ Correspondence should be addressed to Yasunori Kimura; yasunori@is.sci.toho-u.ac.jp
}

Received 24 September 2012; Accepted 28 December 2012

Academic Editor: Jaan Janno

Copyright (c) 2013 Y. Kimura and K. Nakajo. This is an open access article distributed under the Creative Commons Attribution License, which permits unrestricted use, distribution, and reproduction in any medium, provided the original work is properly cited.

We consider the problem of image recovery by the metric projections in a real Banach space. For a countable family of nonempty closed convex subsets, we generate an iterative sequence converging weakly to a point in the intersection of these subsets. Our convergence theorems extend the results proved by Bregman and Crombez.

\section{Introduction}

Let $C_{1}, C_{2}, \ldots, C_{r}$ be nonempty closed convex subsets of a real Hilbert space $H$ such that $\bigcap_{i=1}^{r} C_{i} \neq \emptyset$. Then, the problem of image recovery may be stated as follows: the original unknown image $z$ is known a priori to belong to the intersection of $\left\{C_{i}\right\}_{i=1}^{r}$; given only the metric projections $P_{C_{i}}$ of $H$ onto $C_{i}$ for $i=1,2, \ldots, r$, recover $z$ by an iterative scheme. Such a problem is connected with the convex feasibility problem and has been investigated by a large number of researchers.

Bregman [1] considered a sequence $\left\{x_{n}\right\}$ generated by cyclic projections, that is, $x_{0}=x \in H, x_{1}=P_{C_{1}} x, x_{2}=$ $P_{C_{2}} x_{1}, x_{3}=P_{C_{3}} x_{2}, \ldots, x_{r}=P_{C_{r}} x_{r-1}, x_{r+1}=P_{C_{1}} x_{r}, x_{r+2}=$ $P_{C_{2}} x_{r+1}, \ldots$. It was proved that $\left\{x_{n}\right\}$ converges weakly to an element of $\bigcap_{i=1}^{r} C_{i}$ for an arbitrary initial point $x \in H$.

Crombez [2] proposed a sequence $\left\{y_{n}\right\}$ generated by $y_{0}=$ $y \in H, y_{n+1}=\alpha_{0} y_{n}+\sum_{i=1}^{r} \alpha_{i}\left(y_{n}+\lambda_{i}\left(P_{C_{i}} y_{n}-y_{n}\right)\right)$ for $n=0,1,2, \ldots$, where $0<\alpha_{i}<1$ for all $i=0,1,2, \ldots, r$ with $\sum_{i=0}^{r} \alpha_{i}=1$ and $0<\lambda_{i}<2$ for every $i=1,2, \ldots, r$. It was proved that $\left\{y_{n}\right\}$ converges weakly to an element of $\bigcap_{i=1}^{r} C_{i}$ for an arbitrary initial point $y \in H$.

Later, Kitahara and Takahashi [3] and Takahashi and Tamura [4] dealt with the problem of image recovery by convex combinations of nonexpansive retractions in a uniformly convex Banach space E. Alber [5] took up the problem of image recovery by the products of generalized projections in a uniformly convex and uniformly smooth Banach space $E$ whose duality mapping is weakly sequentially continuous (see also $[6,7]$ ).

On the other hand, using the hybrid projection method proposed by Haugazeau [8] (see also [9-11] and references therein) and the shrinking projection method proposed by Takahashi et al. [12] (see also [13]), Nakajo et al. [14] and Kimura et al. [15] considered this problem by the metric projections and proved convergence of the iterative sequence to a common point of countable nonempty closed convex subsets in a uniformly convex and smooth Banach space $E$ and in a strictly convex, smooth, and reflexive Banach space $E$ having the Kadec-Klee property, respectively. Kohsaka and Takahashi [16] took up this problem by the generalized projections and obtained the strong convergence to a common point of a countable family of nonempty closed convex subsets in a uniformly convex Banach space whose norm is uniformly Gâteaux differentiable (see also [17, 18]). Although these results guarantee the strong convergence, they need to use metric or generalized projections onto different subsets for each step, which are not given in advance.

In this paper, we consider this problem by the metric projections, which are one of the most familiar projections to deal with. The advantage of our results is that we use projections onto the given family of subsets only, to generate 
the iterative scheme. Our convergence theorems extend the results of $[1,2]$ to a Banach space $E$, and they deduce the weak convergence to a common point of a countable family of nonempty closed convex subsets of $E$.

There are a number of results dealing with the image recovery problem from the aspects of engineering using nonlinear functional analysis (see, e.g., [19]). Comparing with these researches, we may say that our approach is more abstract and theoretical; we adopt a general Banach space with several conditions for an underlying space, and therefore, the technique of the proofs can be applied to various mathematical results related to nonlinear problems defined on Banach spaces.

\section{Preliminaries}

Throughout this paper, let $\mathbb{N}$ be the set of all positive integers, $\mathbb{R}$ the set of all real numbers, $E$ a real Banach space with norm $\|\cdot\|$, and $E^{*}$ the dual of $E$. For $x \in E$ and $x^{*} \in E^{*}$, we denote by $\left\langle x, x^{*}\right\rangle$ the value of $x^{*}$ at $x$. We write $x_{n} \rightarrow x$ to indicate that a sequence $\left\{x_{n}\right\}$ converges strongly to $x$. Similarly, $x_{n} \rightarrow x$ and $x_{n} \stackrel{*}{\rightarrow} x$ will symbolize weak and weak ${ }^{*}$ convergence, respectively. We define the modulus $\delta_{E}$ of convexity of $E$ as follows: $\delta_{E}$ is a function of $[0,2]$ into $[0,1]$ such that

$$
\begin{gathered}
\delta_{E}(\epsilon)=\inf \left\{1-\frac{\|x+y\|}{2}: x, y \in E,\|x\|=1,\right. \\
\|y\|=1,\|x-y\| \geq \epsilon\}
\end{gathered}
$$

for every $\epsilon \in[0,2]$. $E$ is called uniformly convex if $\delta_{E}(\epsilon)>0$ for each $\epsilon>0$. Let $p>1$. $E$ is said to be $p$-uniformly convex if there exists a constant $c>0$ such that $\delta_{E}(\epsilon) \geq c \epsilon^{p}$ for every $\epsilon \in[0,2]$. It is obvious that a $p$-uniformly convex Banach space is uniformly convex. $E$ is said to be strictly convex if $\|x+y\| / 2<1$ for all $x, y \in E$ with $\|x\|=\|y\|=1$ and $x \neq y$. We know that a uniformly convex Banach space is strictly convex and reflexive. For every $p>1$, the (generalized) duality mapping $J_{p}: E \rightarrow 2^{E^{*}}$ of $E$ is defined by

$$
J_{p} x=\left\{y^{*} \in E^{*}:\left\langle x, y^{*}\right\rangle=\|x\|^{p},\left\|y^{*}\right\|=\|x\|^{p-1}\right\}
$$

for all $x \in E$. When $p=2, J_{2}$ is called the normalized duality mapping. We have that for $p, q>1,\|x\|^{p} J_{q} x=\|x\|^{q} J_{p} x$ for all $x \in E$. $E$ is said to be smooth if the limit

$$
\lim _{t \rightarrow 0} \frac{\|x+t y\|-\|x\|}{t}
$$

exists for every $x, y \in E$ with $\|x\|=\|y\|=1$. We know that the duality mapping $J_{p}$ of $E$ is single valued for each $p>1$ if $E$ is smooth. It is also known that if $E$ is strictly convex, then the duality mapping $J_{p}$ of $E$ is one to one in the sense that $x \neq y$ implies that $J_{p} x \cap J_{p} y=\emptyset$ for all $p>1$. If $E$ is reflexive, then $J_{p}$ is surjective, and $J_{p}^{-1}$ is identical to the duality mapping $J_{q}^{*}: E^{*} \rightarrow 2^{E}$ defined by

$$
J_{q}^{*} y^{*}=\left\{x \in E:\left\langle x, y^{*}\right\rangle=\left\|y^{*}\right\|^{q},\|x\|=\left\|y^{*}\right\|^{q-1}\right\}
$$

for every $y^{*} \in E^{*}$, where $q \in \mathbb{R}$ satisfies $1 / p+1 / q=1$. For $p>1$, the duality mapping $J_{p}$ of a smooth Banach space $E$ is said to be weakly sequentially continuous if $x_{n} \rightarrow x$ implies that $J_{p} x_{n} \stackrel{*}{\rightarrow} J_{p} x$ (see $[20,21]$ for details). The following is proved by $\mathrm{Xu}[22]$ (see also [23]).

Theorem 1 (Xu [22]). Let $E$ be a smooth Banach space and $p>1$. Then, $E$ is $p$-uniformly convex if and only if there exists a constant $c>0$ such that $\|x+y\|^{p} \geq\|x\|^{p}+p\left\langle y, J_{p} x\right\rangle+c\|y\|^{p}$ holds for every $x, y \in E$.

Remark 2. For a $p$-uniformly convex and smooth Banach space $E$, we have that the constant $c$ in the theorem above satisfies $c \leq 1$. Let

$$
\begin{gathered}
c_{0}=\sup \left\{c>0:\|x+y\|^{p} \geq\|x\|^{p}+p\left\langle y, J_{p} x\right\rangle\right. \\
\left.+c\|y\|^{p} \forall x, y \in E\right\} .
\end{gathered}
$$

Then, there exists a positive real sequence $\left\{c_{n}\right\}$ such that $\lim _{n \rightarrow \infty} c_{n}=c_{0}$ and $\|x+y\|^{p} \geq\|x\|^{p}+p\left\langle y, J_{p} x\right\rangle+c_{n}\|y\|^{p}$ for all $x, y \in E$ and $n \in \mathbb{N}$. So, we get $\|x+y\|^{p} \geq\|x\|^{p}+$ $p\left\langle y, J_{p} x\right\rangle+c_{0}\|y\|^{p}$ for every $x, y \in E$. Therefore, $c_{0}$ is the maximum of constants. In the case of Hilbert spaces, the normalized duality mapping $J_{2}$ is the identity mapping and $c_{0}=1$.

Let $E$ be a smooth Banach space and $p>1$. The function $\phi_{p}: E \times E \rightarrow \mathbb{R}$ is defined by

$$
\phi_{p}(y, x)=\|y\|^{p}-p\left\langle y, J_{p} x\right\rangle+(p-1)\|x\|^{p}
$$

for every $x, y \in E$. We have $\phi_{p}(x, y) \geq 0$ for all $x, y \in E$ and $\phi_{p}(z, x)+\phi_{p}(x, y)=\phi_{p}(z, y)+p\left\langle x-z, J_{p} x-J_{p} y\right\rangle$ for every $x, y, z \in E$. It is known that if $E$ is strictly convex and smooth, then, for $x, y \in E, \phi_{p}(y, x)=\phi_{p}(x, y)=0$ if and only if $x=y$. Indeed, suppose that $\phi_{p}(y, x)=\phi_{p}(x, y)=0$. Then, since

$$
\begin{aligned}
0 & =\phi_{p}(y, x)+\phi_{p}(x, y) \\
& =p\left(\|x\|^{p}+\|y\|^{p}-\left\langle x, J_{p} y\right\rangle-\left\langle y, J_{p} x\right\rangle\right) \\
& \geq p\left(\|x\|^{p}+\|y\|^{p}-\|x\|\|y\|^{p-1}-\|y\|\|x\|^{p-1}\right) \\
& =p(\|x\|-\|y\|)\left(\|x\|^{p-1}-\|y\|^{p-1}\right) \geq 0,
\end{aligned}
$$

we have $\|x\|=\|y\|$. It follows that $\left\langle y, J_{p} x\right\rangle=p^{-1}\left(\|y\|^{p}+(p-\right.$ 1) $\left.\|x\|^{p}-\phi_{p}(y, x)\right)=\|y\|^{p}$ and $\left\|J_{p} x\right\|=\|x\|^{p-1}=\|y\|^{p-1}$, which implies that $J_{p} y=J_{p} x$. Since $J_{p}$ is one to one, we have $x=y$ (see also [17]). We have the following result from Theorem 1 . 
Lemma 3. Let $p>1$ and $E$ be a p-uniformly convex and smooth Banach space. Then, for each $x, y \in E$,

$$
\phi_{p}(x, y) \geq c_{0}\|x-y\|^{p}
$$

holds, where $c_{0}$ is maximum in Remark 2.

Proof. Let $x, y \in E$. By Theorem 1, we have

$$
\|x\|^{p} \geq\|y\|^{p}+p\left\langle x-y, J_{p} y\right\rangle+c_{0}\|x-y\|^{p},
$$

where $c_{0}$ is maximum in Remark 2 . Hence, we get

$$
\begin{aligned}
\phi_{p}(x, y) & =\|x\|^{p}-\|y\|^{p}-p\left\langle x-y, J_{p} y\right\rangle \\
& \geq c_{0}\|x-y\|^{p},
\end{aligned}
$$

which is the desired result.

Let $C$ be a nonempty closed convex subset of a strictly convex and reflexive Banach space $E$, and let $x \in E$. Then, there exists a unique element $x_{0} \in C$ such that $\left\|x_{0}-x\right\|=$ inf $_{y \in C}\|y-x\|$. Putting $x_{0}=P_{C} x$, we call $P_{C}$ the metric projection onto $C$ (see [24]). We have the following result [25, p. 196] for the metric projection.

Lemma 4. Let $C$ be a nonempty closed convex subset of a strictly convex, reflexive, and smooth Banach space $E$, and let $x \in E$. Then, $y=P_{C} x$ if and only if $\left\langle y-z, J_{2}(x-y)\right\rangle \geq 0$ for all $z \in C$, where $P_{C}$ is the metric projection onto $C$.

Remark 5. For $p>1$, it holds that $\|x\| J_{p} x=\|x\|^{p-1} J_{2} x$ for every $x \in E$. Therefore, under the same assumption as Lemma 4, we have that $y=P_{C} x$ if and only if $\left\langle y-z, J_{p}(x-\right.$ $y)\rangle \geq 0$ for all $z \in C$.

\section{Main Results}

Firstly, we consider the iteration of Crombez's type and get the following result.

Theorem 6. Let $p, q>1$ be such that $1 / p+1 / q=1$. Let $\left\{C_{n}\right\}_{n \in \mathbb{N}}$ be a family of nonempty closed convex subsets of a $p$ uniformly convex and smooth Banach space $E$ whose duality mapping $J_{p}$ is weakly sequentially continuous. Suppose that $\bigcap_{n \in \mathbb{N}} C_{n} \neq \emptyset$. Let $\left.\lambda_{n, k} \in\right] 0,(1+1 /(p-1))^{p-1} c_{0}\left[\right.$ and $\alpha_{n, k} \in[0,1]$ for all $n \in \mathbb{N}$ and $k=1,2, \ldots, n$ with $\sum_{k=1}^{n} \alpha_{n, k}=1$ for every $n \in \mathbb{N}$, where $c_{0}$ is maximum in Remark 2. Let $\left\{x_{n}\right\}$ be a sequence generated by $x_{1}=x \in E$ and

$$
x_{n+1}=J_{q}^{*}\left(\sum_{k=1}^{n} \alpha_{n, k}\left(J_{p} x_{n}-\lambda_{n, k} J_{p}\left(x_{n}-P_{C_{k}} x_{n}\right)\right)\right)
$$

for every $n \in \mathbb{N}$. If $0<\liminf _{n \rightarrow \infty} \lambda_{n, k} \leq \limsup _{n \rightarrow \infty} \lambda_{n, k}<$ $(1+1 /(p-1))^{p-1} c_{0}$ and $\liminf _{n \rightarrow \infty} \alpha_{n, k}>0$ for each $k \in \mathbb{N}$, then $\left\{x_{n}\right\}$ converges weakly to a point in $\bigcap_{n=1}^{\infty} C_{n}$.
Proof. Let $y_{n, k}=J_{q}^{*}\left(J_{p} x_{n}-\lambda_{n, k} J_{p}\left(x_{n}-P_{C_{k}} x_{n}\right)\right)$ for $n \in \mathbb{N}$ and $k=1,2, \ldots, n$. Then, for $z \in \bigcap_{n \in \mathbb{N}} C_{n}$, we obtain

$$
\begin{aligned}
\phi_{p}\left(z, y_{n, k}\right)-\phi_{p}\left(z, x_{n}\right) \\
=-\phi_{p}\left(y_{n, k}, x_{n}\right)+p\left\langle y_{n, k}-z, J_{p} y_{n, k}-J_{p} x_{n}\right\rangle \\
=-\phi_{p}\left(y_{n, k}, x_{n}\right)-p \lambda_{n, k}\left\langle y_{n, k}-z, J_{p}\left(x_{n}-P_{C_{k}} x_{n}\right)\right\rangle \\
=-\phi_{p}\left(y_{n, k}, x_{n}\right)-p \lambda_{n, k}\left\langle y_{n, k}-x_{n}, J_{p}\left(x_{n}-P_{C_{k}} x_{n}\right)\right\rangle \\
\quad-p \lambda_{n, k}\left\langle x_{n}-z, J_{p}\left(x_{n}-P_{C_{k}} x_{n}\right)\right\rangle
\end{aligned}
$$

for all $n \in \mathbb{N}$ and $k=1,2, \ldots, n$. Using Remark 5 with that $z \in C_{k}$, we get

$$
\begin{aligned}
\left\langle x_{n}-z, J_{p}\left(x_{n}-P_{C_{k}} x_{n}\right)\right\rangle & \\
= & \left\langle x_{n}-P_{C_{k}} x_{n}, J_{p}\left(x_{n}-P_{C_{k}} x_{n}\right)\right\rangle \\
& +\left\langle P_{C_{k}} x_{n}-z, J_{p}\left(x_{n}-P_{C_{k}} x_{n}\right)\right\rangle \\
\geq & \left\|x_{n}-P_{C_{k}} x_{n}\right\|^{p}
\end{aligned}
$$

for every $n \in \mathbb{N}$ and $k=1,2 \ldots, n$. Thus, by Lemma 3 we have

$$
\begin{aligned}
\phi_{p}\left(z, y_{n, k}\right)-\phi_{p}\left(z, x_{n}\right) \\
\leq-c_{0}\left\|y_{n, k}-x_{n}\right\|^{p} \\
\quad-p \lambda_{n, k}\left\langle y_{n, k}-x_{n}, J_{p}\left(x_{n}-P_{C_{k}} x_{n}\right)\right\rangle \\
\quad-p \lambda_{n, k}\left\|x_{n}-P_{C_{k}} x_{n}\right\|^{p} \\
\leq-c_{0}\left\|y_{n, k}-x_{n}\right\|^{p}+p \lambda_{n, k}\left\|y_{n, k}-x_{n}\right\|\left\|x_{n}-P_{C_{k}} x_{n}\right\|^{p-1} \\
\quad-p \lambda_{n, k}\left\|x_{n}-P_{C_{k}} x_{n}\right\|^{p}
\end{aligned}
$$

for each $n \in \mathbb{N}$ and $k=1,2, \ldots, n$. Since it holds that

$$
s t \leq \frac{1}{\beta} \frac{s^{p}}{p}+\beta^{q-1} \frac{t^{q}}{q}
$$

for $s, t \geq 0, p, q>1$ with $1 / p+1 / q=1$, and $\beta>0$, we have

$$
\begin{aligned}
\| y_{n, k}- & x_{n}\|\| x_{n}-P_{C_{k}} x_{n} \|^{p-1} \\
\leq & \frac{1}{\beta_{k} p}\left\|y_{n, k}-x_{n}\right\|^{p} \\
& +\beta_{k}^{1 /(p-1)} \frac{p-1}{p}\left\|x_{n}-P_{C_{k}} x_{n}\right\|^{p}
\end{aligned}
$$

for every $k \in \mathbb{N}, \beta_{k}>0$ and $n \geq k$. Therefore, it follows that

$$
\begin{aligned}
\phi_{p}\left(z, y_{n, k}\right)-\phi_{p}\left(z, x_{n}\right) & \\
\leq & \left(\frac{\lambda_{n, k}}{\beta_{k}}-c_{0}\right)\left\|y_{n, k}-x_{n}\right\|^{p} \\
& +\lambda_{n, k}\left((p-1) \beta_{k}^{1 /(p-1)}-p\right)\left\|x_{n}-P_{C_{k}} x_{n}\right\|^{p}
\end{aligned}
$$


for every $n \in \mathbb{N}, k=1,2, \ldots, n$, and $\beta_{k}>0$. Since

$$
\begin{aligned}
\phi_{p}\left(z, x_{n+1}\right)= & \|z\|^{p}-p\left\langle z, \sum_{k=1}^{n} \alpha_{n, k} J_{p} y_{n, k}\right\rangle \\
& +(p-1)\left\|\sum_{k=1}^{n} \alpha_{n, k} J_{p} y_{n, k}\right\|^{p /(p-1)} \\
\leq & \|z\|^{p}-p \sum_{k=1}^{n} \alpha_{n, k}\left\langle z, J_{p} y_{n, k}\right\rangle \\
& +(p-1) \sum_{k=1}^{n} \alpha_{n, k}\left\|y_{n, k}\right\|^{p} \\
= & \sum_{k=1}^{n} \alpha_{n, k} \phi_{p}\left(z, y_{n, k}\right)
\end{aligned}
$$

for every $n \in \mathbb{N}$, we have

$$
\begin{aligned}
\phi_{p}\left(z, x_{n+1}\right)-\phi_{p}\left(z, x_{n}\right) \\
\leq \sum_{k=1}^{n} \alpha_{n, k}\left(\frac{\lambda_{n, k}}{\beta_{k}}-c_{0}\right)\left\|y_{n, k}-x_{n}\right\|^{p} \\
\quad+\sum_{k=1}^{n} \alpha_{n, k} \lambda_{n, k}\left((p-1) \beta_{k}^{1 /(p-1)}-p\right)\left\|x_{n}-P_{C_{k}} x_{n}\right\|^{p}
\end{aligned}
$$

for all $n \in \mathbb{N}$ and $\beta_{1}, \beta_{2}, \ldots, \beta_{n}>0$. Since $\left.\lambda_{n, k} \in\right] 0,(1+1 /(p-$ 1)) $)^{p-1} c_{0}\left[, \alpha_{n, k} \in[0,1]\right.$ for all $n \in \mathbb{N}$ and $k=1,2, \ldots, n$,

$$
\begin{gathered}
0<\liminf _{n \rightarrow \infty} \lambda_{n, k} \leq \limsup _{n \rightarrow \infty} \lambda_{n, k}<\left(1+\frac{1}{(p-1)}\right)^{p-1} c_{0}, \\
\liminf _{n \rightarrow \infty} \alpha_{n, k}>0
\end{gathered}
$$

for each $k \in \mathbb{N}$, we can choose $\beta_{k}>0$ for every $k \in \mathbb{N}$ such that $\alpha_{n, k}\left(\lambda_{n, k} / \beta_{k}-c_{0}\right) \leq 0, \alpha_{n, k} \lambda_{n, k}\left((p-1) \beta_{k}^{1 /(p-1)}-p\right) \leq 0$ for all $n \geq k$ and

$$
\begin{gathered}
\limsup _{n \rightarrow \infty} \alpha_{n, k}\left(\frac{\lambda_{n, k}}{\beta_{k}}-c_{0}\right)<0 \\
\limsup _{n \rightarrow \infty} \alpha_{n, k} \lambda_{n, k}\left((p-1) \beta_{k}^{1 /(p-1)}-p\right)<0
\end{gathered}
$$

for each $k \in \mathbb{N}$. Hence, there exists $\lim _{n \rightarrow \infty} \phi_{p}\left(z, x_{n}\right)$ for every $z \in \bigcap_{n \in \mathbb{N}} C_{n}$ and

$$
\lim _{n \rightarrow \infty}\left\|y_{n, k}-x_{n}\right\|=\lim _{n \rightarrow \infty}\left\|x_{n}-P_{C_{k}} x_{n}\right\|=0
$$

for all $k \in \mathbb{N}$. It follows from Lemma 3 that $\left\{x_{n}\right\}$ is bounded. Let $\left\{x_{n_{i}}\right\}$ and $\left\{x_{m_{j}}\right\}$ be subsequences of $\left\{x_{n}\right\}$ such that $x_{n_{i}} \rightarrow u_{1}$ and $x_{m_{j}} \rightarrow u_{2}$. Then, we get $\left\|x_{n_{i}}-P_{C_{k}} x_{n_{i}}\right\| \rightarrow 0$ which implies that $u_{1} \in C_{k}$ for every $k \in \mathbb{N}$. In the same way, we also have $u_{2} \in C_{k}$ for every $k \in \mathbb{N}$. Let $\lim _{n \rightarrow \infty} \phi_{p}\left(u_{1}, x_{n}\right)=\mu_{1}$ and $\lim _{n \rightarrow \infty} \phi_{p}\left(u_{2}, x_{n}\right)=\mu_{2}$. Since

$$
\begin{aligned}
\mu_{1}-\mu_{2} & =\lim _{i \rightarrow \infty}\left(\phi_{p}\left(u_{1}, x_{n_{i}}\right)-\phi_{p}\left(u_{2}, x_{n_{i}}\right)\right) \\
& =\left\|u_{1}\right\|^{p}-\left\|u_{2}\right\|^{p}+p \lim _{i \rightarrow \infty}\left\langle u_{2}-u_{1}, J_{p} x_{n_{i}}\right\rangle
\end{aligned}
$$

and $J_{p}$ is weakly sequentially continuous, we have

$$
\begin{aligned}
\mu_{1}-\mu_{2} & =\left\|u_{1}\right\|^{p}-\left\|u_{2}\right\|^{p}+p\left\langle u_{2}-u_{1}, J_{p} u_{1}\right\rangle \\
& =-\phi_{p}\left(u_{2}, u_{1}\right) .
\end{aligned}
$$

Similarly, we obtain $\mu_{2}-\mu_{1}=-\phi_{p}\left(u_{1}, u_{2}\right)$. So, we get $\phi_{p}\left(u_{1}, u_{2}\right)+\phi_{p}\left(u_{2}, u_{1}\right)=0$, that is, $u_{1}=u_{2}$. Therefore, $\left\{x_{n}\right\}$ converges weakly to a point in $\bigcap_{n \in \mathbb{N}} C_{n}$.

Using the idea of $[9$, p. 256], we also have the following result by the iteration of Bregman's type.

Theorem 7. Let $p, q>1$ be such that $1 / p+1 / q=1$. Let $I$ be a countable set and $\left\{C_{j}\right\}_{j \in I}$ a family of nonempty closed convex subsets of a p-uniformly convex and smooth Banach space E whose duality mapping $J_{p}$ is weakly sequentially continuous. Suppose that $\bigcap_{j \in I} C_{j} \neq \emptyset$. Let $\left.\lambda_{n} \in\right] 0,(1+1 /(p-1))^{p-1} c_{0}[$ for all $n \in \mathbb{N}$, where $c_{0}$ is maximum in Remark 2 , and let $\left\{x_{n}\right\}$ be a sequence generated by $x_{1}=x \in E$ and

$$
x_{n+1}=J_{q}^{*}\left(J_{p} x_{n}-\lambda_{n} J_{p}\left(x_{n}-P_{C_{i(n)}} x_{n}\right)\right)
$$

for every $n \in \mathbb{N}$, where the index mapping $i: \mathbb{N} \rightarrow$ I satisfies that, for every $j \in I$, there exists $M_{j} \in \mathbb{N}$ such that $j \in$ $\left\{i(n), \ldots, i\left(n+M_{j}-1\right)\right\}$ for each $n \in \mathbb{N}$. If $0<\liminf _{n \rightarrow \infty} \lambda_{n} \leq$ $\limsup _{n \rightarrow \infty} \lambda_{n}<(1+1 /(p-1))^{p-1} c_{0}$, then, $\left\{x_{n}\right\}$ converges weakly to a point in $\bigcap_{j \in I} C_{j}$.

Proof. Let $z \in \bigcap_{j \in I} C_{j}$. As in the proof of Theorem 6, we have

$$
\begin{aligned}
\phi_{p}\left(z, x_{n+1}\right)-\phi_{p}\left(z, x_{n}\right) & \\
\leq & \left(\frac{\lambda_{n}}{\beta}-c_{0}\right)\left\|x_{n+1}-x_{n}\right\|^{p} \\
& +\lambda_{n}\left((p-1) \beta^{1 /(p-1)}-p\right)\left\|x_{n}-P_{C_{i(n)}} x_{n}\right\|^{p}
\end{aligned}
$$

for all $n \in \mathbb{N}$ and $\beta>0$. Since $\left.\lambda_{n} \in\right] 0,(1+1 /(p-1))^{p-1} c_{0}[$ for all $n \in \mathbb{N}$ and $0<\liminf _{n \rightarrow \infty} \lambda_{n} \leq \limsup _{n \rightarrow \infty} \lambda_{n}<$ $(1+1 /(p-1))^{p-1} c_{0}$, we can find that $\beta>0$ such that

$$
\limsup _{n \rightarrow \infty}\left(\frac{\lambda_{n}}{\beta}-c_{0}\right)<0,
$$

$$
\limsup _{n \rightarrow \infty} \lambda_{n}\left((p-1) \beta^{1 /(p-1)}-p\right)<0 \text {. }
$$

Then, there exists $\lim _{n \rightarrow \infty} \phi_{p}\left(z, x_{n}\right)$ for every $z \in \bigcap_{i \in I} C_{i}$ and

$$
\lim _{n \rightarrow \infty}\left\|x_{n+1}-x_{n}\right\|=\lim _{n \rightarrow \infty}\left\|x_{n}-P_{C_{i(n)}} x_{n}\right\|=0 .
$$


So, we have that $\left\{x_{n}\right\}$ is bounded from Lemma 3. Let $\left\{x_{n_{k}}\right\}$ be a subsequence of $\left\{x_{n}\right\}$ such that $x_{n_{k}} \rightarrow u$. For fixed $j \in I$, there exists a strictly increasing sequence $\left\{m_{k}\right\} \subset \mathbb{N}$ such that $n_{k} \leq m_{k} \leq n_{k}+M_{j}-1$ and $i\left(m_{k}\right)=j$ for every $k \in \mathbb{N}$. It follows that

$$
\left\|x_{m_{k}}-x_{n_{k}}\right\| \leq \sum_{l=n_{k}}^{n_{k}+M_{j}-1}\left\|x_{l+1}-x_{l}\right\|
$$

for all $k \in \mathbb{N}$ which implies that $x_{m_{k}} \rightarrow u$. Since $\lim _{k \rightarrow \infty}\left\|x_{m_{k}}-P_{C_{j}} x_{m_{k}}\right\|=0, u \in C_{j}$ for every $j \in I$. So, we get $u \in \bigcap_{j \in I} C_{j}$. As in the proof of Theorem 6 , using that $J_{p}$ is weakly sequentially continuous, we get that $\left\{x_{n}\right\}$ converges weakly to a point in $\bigcap_{j \in I} C_{j}$.

Suppose that the index set $I$ is a finite set $\{0,1,2, \ldots, N-$ $1\}$. For the cyclic iteration, the index mapping $i$ is defined by $i(j)=j \bmod N$ for each $j \in I$. Clearly it satisfies the assumption in Theorem 7. In the case where the index set $I$ is countably infinite, that is, $I=\mathbb{N}$, one of the simplest examples of $i: \mathbb{N} \rightarrow \mathbb{N}$ can be defined as follows:

$$
i(n)= \begin{cases}1 & (n=2 m-1 \text { for some } m \in \mathbb{N}), \\ 2 & (n=2(2 m-1) \text { for some } m \in \mathbb{N}), \\ 3 & (n=4(2 m-1) \text { for some } m \in \mathbb{N}), \\ k & \left(n=2^{k-1}(2 m-1) \text { for some } m \in \mathbb{N}\right), \\ \ldots & \end{cases}
$$

Then, the assumption in Theorem 7 is satisfied by letting $M_{j}=2^{j}$ for each $j \in I=\mathbb{N}$.

\section{Deduced Results}

Since a real Hilbert space $H$ is 2-uniformly convex and the maximum $c_{0}$ in Remark 2 is equal to 1 , we get the following results. At first, we have the following theorem which generalizes the results of [2] by Theorem 6 .

Theorem 8. Let $\left\{C_{n}\right\}_{n \in \mathbb{N}}$ be a family of nonempty closed convex subsets of $H$ such that $\bigcap_{n \in \mathbb{N}} C_{n} \neq \emptyset$. Let $\left.\lambda_{n, k} \in\right] 0,2\left[\right.$ and $\alpha_{n, k} \in$ $[0,1]$ for all $n \in \mathbb{N}$ and $k=1,2, \ldots, n$ with $\sum_{k=1}^{n} \alpha_{n, k}=1$ for every $n \in \mathbb{N}$. Let $\left\{x_{n}\right\}$ be a sequence generated by $x_{1}=x \in H$ and

$$
x_{n+1}=\sum_{k=1}^{n} \alpha_{n, k}\left(x_{n}-\lambda_{n, k}\left(x_{n}-P_{C_{k}} x_{n}\right)\right)
$$

for every $n \in \mathbb{N}$. If it holds that $0<\liminf _{n \rightarrow \infty} \lambda_{n, k} \leq$ $\lim \sup _{n \rightarrow \infty} \lambda_{n, k}<2$ and $\lim \inf _{n \rightarrow \infty} \alpha_{n, k}>0$ for each $k \in \mathbb{N}$, then, $\left\{x_{n}\right\}$ converges weakly to a point in $\bigcap_{n=1}^{\infty} C_{n}$.

Next, we have the following theorem which extends the result of [1] by Theorem 7 .

Theorem 9. Let I be a countable set and $\left\{C_{j}\right\}_{j \in I}$ a family of nonempty closed convex subsets of $H$ such that $\bigcap_{j \in I} C_{j} \neq \emptyset$. Let $\left.\lambda_{n} \in\right] 0,2\left[\right.$ for all $n \in \mathbb{N}$, and let $\left\{x_{n}\right\}$ be a sequence generated by $x_{1}=x \in H$ and

$$
x_{n+1}=x_{n}-\lambda_{n}\left(x_{n}-P_{C_{i(n)}} x_{n}\right)
$$

for every $n \in \mathbb{N}$, where the index mapping $i: \mathbb{N} \rightarrow$ I satisfies that, for every $j \in I$, there exists $M_{j} \in \mathbb{N}$ such that $j \in$ $\left\{i(n), \ldots, i\left(n+M_{j}-1\right)\right\}$ for each $n \in \mathbb{N}$. If $0<\liminf _{n \rightarrow \infty} \lambda_{n} \leq$ limsup $\operatorname{sum}_{n \rightarrow \infty} \lambda_{n}<2$, then, $\left\{x_{n}\right\}$ converges weakly to a point in $\bigcap_{j \in I} C_{j}$.

\section{Acknowledgment}

The first author was supported by the Grant-in-Aid for Scientific Research no. 22540175 from the Japan Society for the Promotion of Science.

\section{References}

[1] L. M. Bregman, "The method of successive projection for finding a common point of convexsets," Soviet Mathematics, vol. 6, pp. 688-692, 1965.

[2] G. Crombez, "Image recovery by convex combinations of projections," Journal of Mathematical Analysis and Applications, vol. 155, no. 2, pp. 413-419, 1991.

[3] S. Kitahara and W. Takahashi, "Image recovery by convex combinations of sunny nonexpansive retractions," Topological Methods in Nonlinear Analysis, vol. 2, no. 2, pp. 333-342, 1993.

[4] W. Takahashi and T. Tamura, "Limit theorems of operators by convex combinations of nonexpansive retractions in Banach spaces," Journal of Approximation Theory, vol. 91, no. 3, pp. 386397, 1997.

[5] Y. I. Alber, "Metric and generalized projections operators in Banach spaces: properties andapplications," in Theory and Applications of Nonlinear Operators of Accretive and Monotone Type, A. G. Kartasatos, Ed., vol. 178 of Lecture Notes in Pure and Applied Mathematics, pp. 15-50, Marcel Dekker, New York, NY, USA, 1996.

[6] Y. I. Alber and S. Reich, "An iterative method for solving a class of nonlinear operator equations in Banach spaces," Panamerican Mathematical Journal, vol. 4, no. 2, pp. 39-54, 1994.

[7] S. Kamimura and W. Takahashi, "Strong convergence of a proximal-type algorithm in a Banach space," SIAM Journal on Optimization, vol. 13, no. 3, pp. 938-945, 2002.

[8] Y. Haugazeau, Sur les inéquations variationnelles et la minimisation de fonctionnelles convexes, [Thése], These, Universit é de Paris, Paris, France, 1968.

[9] H. H. Bauschke and P. L. Combettes, "A weak-to-strong convergence principle for Fejér-monotone methods in Hilbert spaces," Mathematics of Operations Research, vol. 26, no. 2, pp. 248-264, 2001.

[10] K. Nakajo and W. Takahashi, "Strong convergence theorems for nonexpansive mappings and nonexpansive semigroups," Journal of Mathematical Analysis and Applications, vol. 279, no. 2, pp. 372-379, 2003.

[11] S. Ohsawa and W. Takahashi, "Strong convergence theorems for resolvents of maximal monotone operators in Banach spaces," Archiv der Mathematik, vol. 81, no. 4, pp. 439-445, 2003. 
[12] W. Takahashi, Y. Takeuchi, and R. Kubota, "Strong convergence theorems by hybrid methods for families of nonexpansive mappings in Hilbert spaces," Journal of Mathematical Analysis and Applications, vol. 341, no. 1, pp. 276-286, 2008.

[13] Y. Kimura and W. Takahashi, "On a hybrid method for a family of relatively nonexpansive mappings in a Banach space," Journal of Mathematical Analysis and Applications, vol. 357, no. 2, pp. 356-363, 2009.

[14] K. Nakajo, K. Shimoji, and W. Takahashi, "Strong convergence theorems by the hybrid method for families of mappings in Banach spaces," Nonlinear Analysis: Theory, Methods \& Applications, vol. 71, no. 3-4, pp. 812-818, 2009.

[15] Y. Kimura, K. Nakajo, and W. Takahashi, "Strongly convergent iterative schemes for a sequence of nonlinear mappings," Journal of Nonlinear and Convex Analysis, vol. 9, no. 3, pp. 407-416, 2008.

[16] F. Kohsaka and W. Takahashi, "Iterative scheme for finding a common point of infinitely many convex sets in a Banach space," Journal of Nonlinear and Convex Analysis, vol. 5, no. 3, pp. 407414, 2004.

[17] S.-y. Matsushita and W. Takahashi, "A strong convergence theorem for relatively nonexpansive mappings in a Banach space," Journal of Approximation Theory, vol. 134, no. 2, pp. 257266, 2005.

[18] S.-y. Matsushita, K. Nakajo, and W. Takahashi, "Strong convergence theorems obtained by a generalized projections hybrid method for families of mappings in Banach spaces," Nonlinear Analysis: Theory, Methods \& Applications A, vol. 73, no. 6, pp. 1466-1480, 2010.

[19] X. Liu and L. Huang, "Total bounded variation-based Poissonian images recovery by split Bregman iteration," Mathematical Methods in the Applied Sciences, vol. 35, no. 5, pp. 520-529, 2012.

[20] I. Cioranescu, Geometry of Banach Spaces, Duality Mappings and Nonlinear Problems, vol. 62 of Mathematics and its Applications, Kluwer Academic Publishers, Dordrecht, The Netherlands, 1990.

[21] J. Diestel, Geometry of Banach Spaces, Selected Topics, vol. 485 of Lecture Notes in Mathematics, Springer, Berlin, Germany, 1975.

[22] H. K. Xu, "Inequalities in Banach spaces with applications," Nonlinear Analysis: Theory, Methods \& Applications, vol. 16, no. 12, pp. 1127-1138, 1991.

[23] B. Beauzamy, Introduction to Banach Spaces and Their Geometry, vol. 68 of North-Holland Mathematics Studies, NorthHolland Publishing, Amsterdam, The Netherlands, 2nd edition, 1985.

[24] K. Goebel and S. Reich, Uniform Convexity, Hyperbolic Geometry, and Nonexpansive Mappings, vol. 83 of Pure and Applied Mathematics, Marcel Dekker, New York, NY, USA, 1984.

[25] W. Takahashi, Nonlinear Functional Analysis, Yokohama Publishers, 2000. 


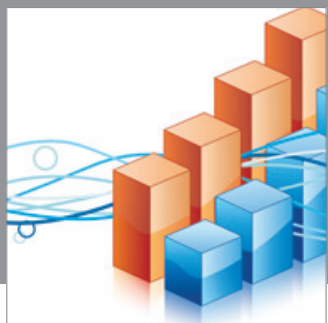

Advances in

Operations Research

mansans

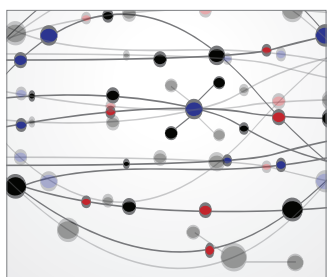

The Scientific World Journal
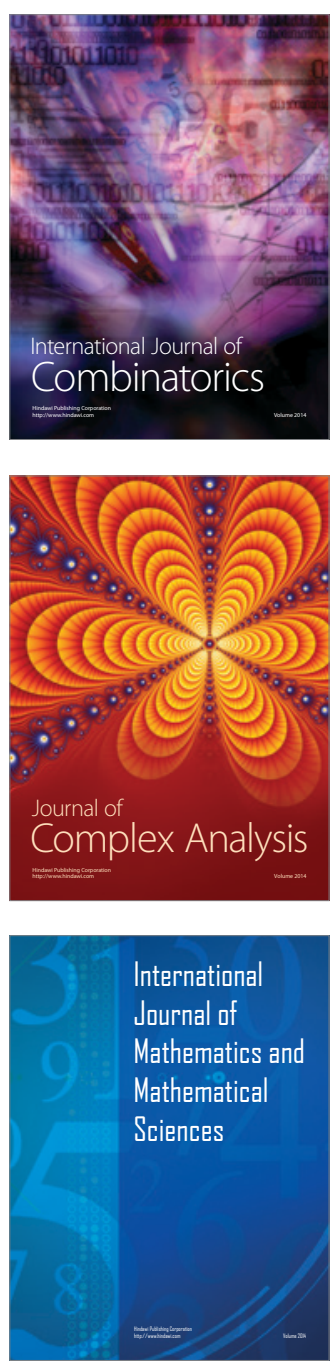
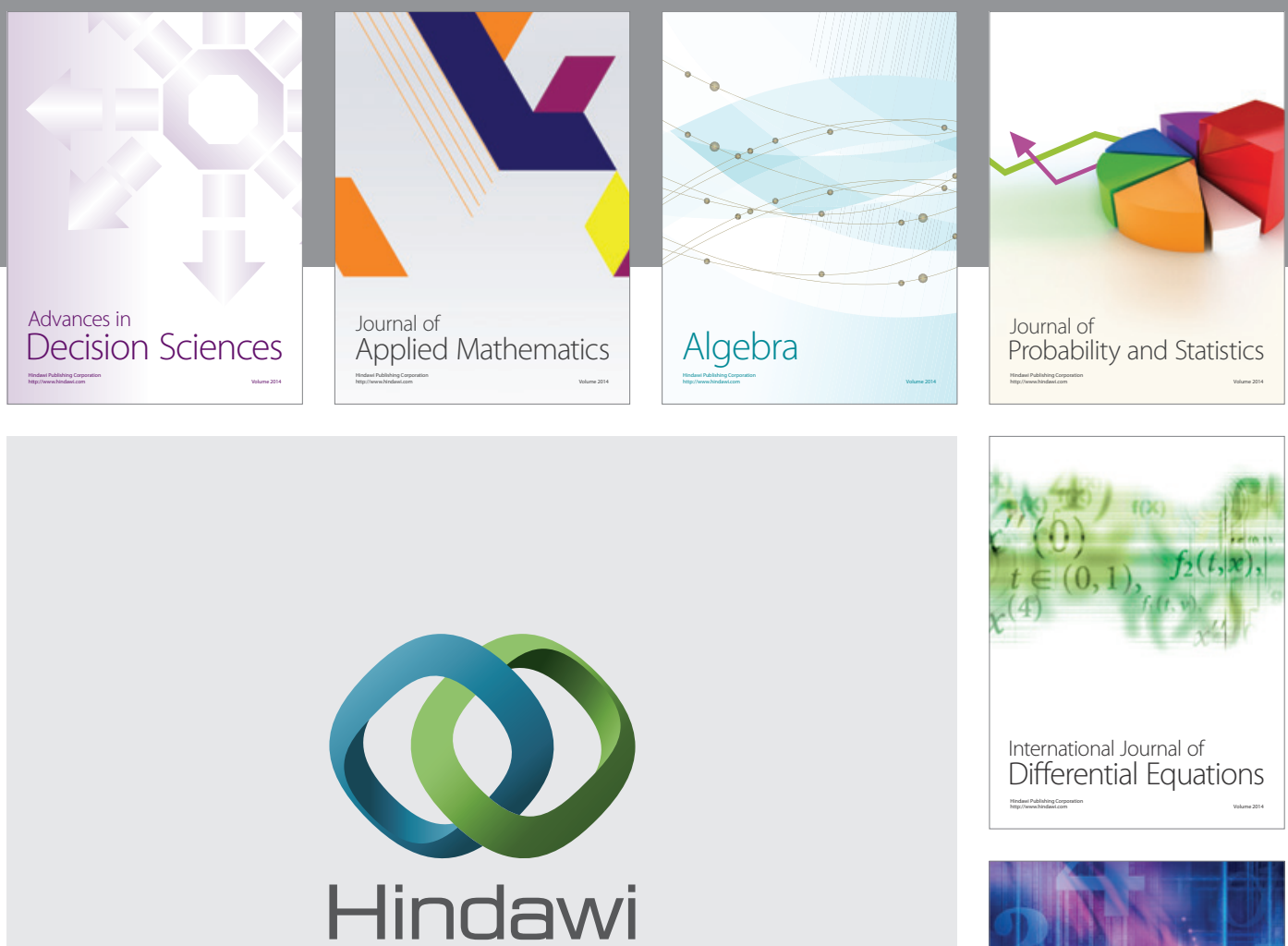

Submit your manuscripts at http://www.hindawi.com
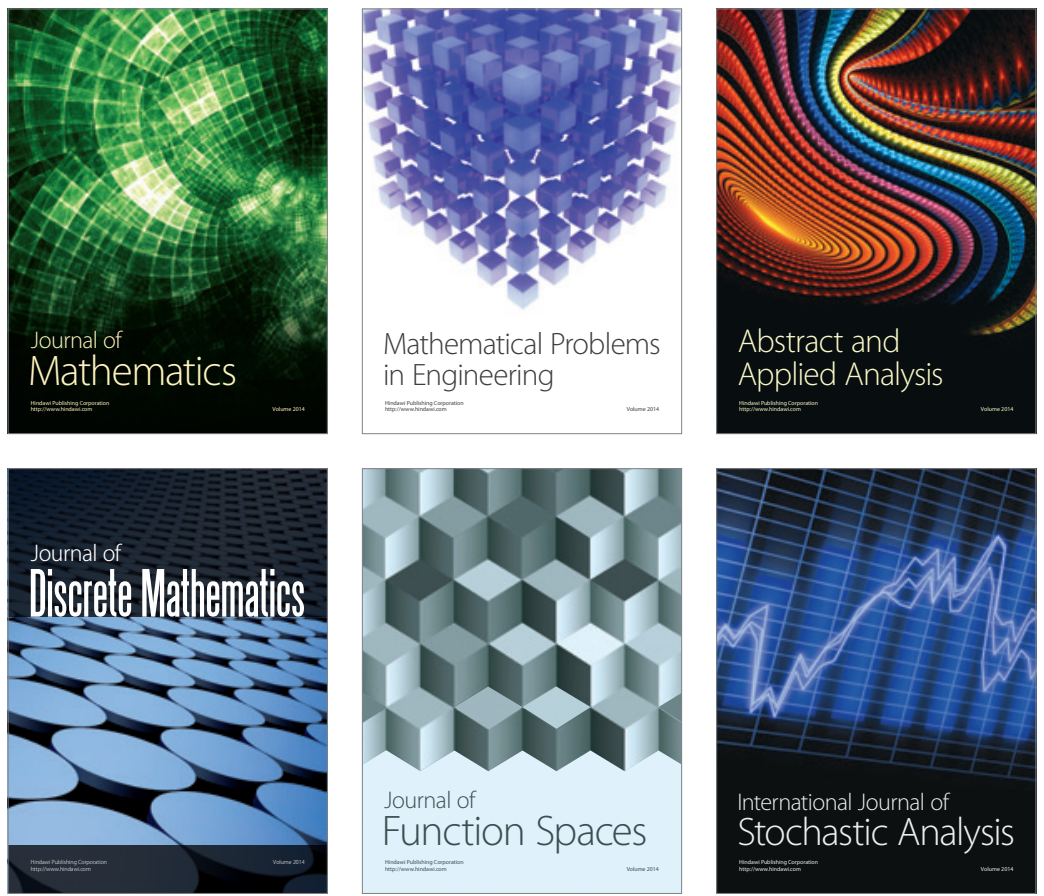

Journal of

Function Spaces

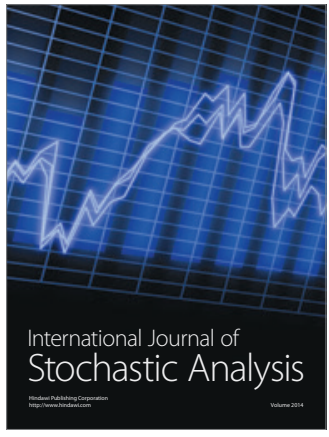

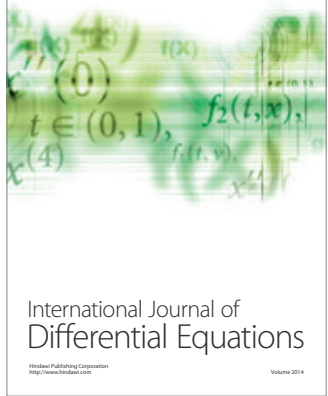
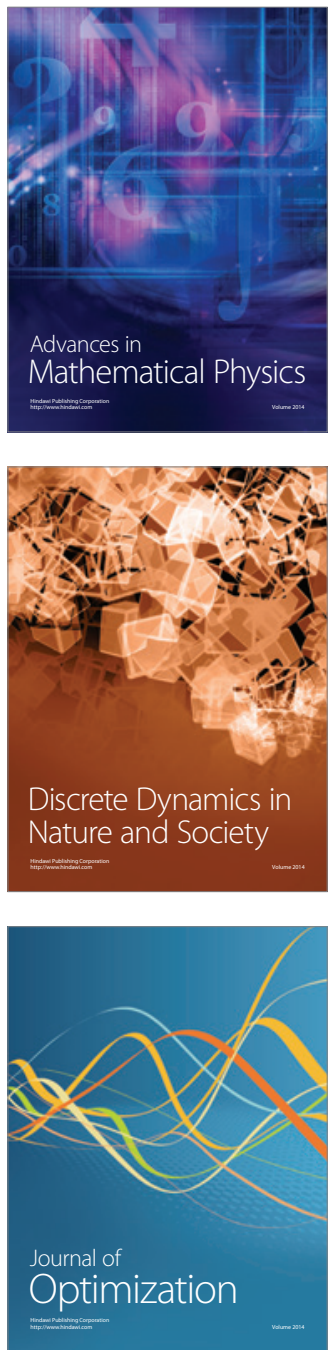